\title{
Sustained activation of the mammalian target of rapamycin nutrient sensing pathway is associated with hepatic insulin resistance, but not with steatosis, in mice
}

\author{
E. Korsheninnikova • G. C. M. van der Zon • \\ P. J. Voshol • G. M. Janssen • L. M. Havekes • \\ A. Grefhorst • F. Kuipers • D.-J. Reijngoud • \\ J. A. Romijn • D. M. Ouwens • J. A. Maassen
}

Received: 10 May 2006 / Accepted: 24 July 2006 / Published online: 28 September 2006

(C) Springer-Verlag 2006

\begin{abstract}
Aims/hypothesis Activation of nutrient sensing through mammalian target of rapamycin (mTOR) has been linked to the pathogenesis of insulin resistance. We examined activation of mTOR-signalling in relation to insulin resistance and hepatic steatosis in mice.

Materials and methods Chronic hepatic steatosis and hepatic insulin resistance were induced by high-fat feeding
\end{abstract}

E. Korsheninnikova $(\bowtie) \cdot$ G. C. M. van der Zon • G. M. Janssen •

D. M. Ouwens $\cdot$ J. A. Maassen

Department of Molecular Cell Biology,

Leiden University Medical Centre,

Postzone S1-P, Postbus 9600,

2300 RC Leiden, the Netherlands

e-mail: Elena@lumc.nl

P. J. Voshol · J. A. Romijn

Department of Endocrinology, Leiden University Medical Centre,

Leiden, the Netherlands

L. M. Havekes

Department of Cardiology and Metabolic Diseases,

Leiden University Medical Centre,

Leiden, the Netherlands

L. M. Havekes

TNO Quality of Life,

Leiden, the Netherlands

A. Grefhorst · F. Kuipers · D.-J. Reijngoud

Center for Liver, Digestive, and Metabolic Diseases,

University Medical Center Groningen,

Groningen, the Netherlands

J. A. Maassen

Department of Endocrinology/Diabetes Center,

VU Medical Center,

Amsterdam, the Netherlands of male C57BL/6Jico mice for 6 weeks. In addition, acute hepatic steatosis in the absence of insulin resistance was induced by pharmacological blockade of $\beta$-oxidation using tetradecylglycidic acid (TDGA). mTOR signalling was examined in liver homogenates.

Results High-fat feeding caused obesity $(p<0.001)$, hepatic steatosis $(p<0.05)$ and hepatic insulin resistance $(p<0.05)$. The phosphorylation of mTOR and its downstream targets p70S6 kinase and S6 ribosomal protein was two-fold higher in mice on a high-fat diet than in mice fed standard chow (all $p<0.05$ ) and associated with enhanced rates of protein synthesis. Acute induction of hepatic steatosis with TDGA had no effect on mTOR activity. The increased activity of the mTOR pathway in livers from mice on a high-fat diet could not be ascribed to diet-induced alterations in known modulators of mTOR activity such as circulating plasma leucine levels, phosphorylation of protein kinase B and AMP-activated protein kinase, and changes in mitochondrial function.

Conclusions/interpretation High-fat diet induces increase of the mTOR nutrient sensing pathway in association with hepatic insulin resistance, but not with hepatic lipid accumulation as such.

Keywords High-fat feeding $\cdot$ Hepatic steatosis · Insulin resistance $\cdot$ Nutrient sensing
Abbreviations
AMPK AMP-activated protein kinase
CPT carnitine palmitoyltransferase
IR insulin receptor
mtDNA mitochondrial DNA
mTOR mammalian target of rapamycin
PI3K phosphatidylinositol 3-kinase 
$\mathrm{PKB} / \mathrm{Akt}$ protein kinase B

TDGA tetradecylglycidic acid

UCP uncoupling protein

\section{Introduction}

The liver is strongly involved in metabolic adaptations in response to variations in the amount and composition of ingested food. Caloric intake in excess of energy expenditure results in obesity and whole-body insulin resistance. For instance, in patients with type 2 diabetes mellitus, hepatic fat content correlates inversely with hepatic insulin sensitivity [1], i.e. the ability of physiological concentrations of insulin to suppress hepatic glucose output, which contributes to the fasting hyperglycaemia observed in these patients [2, 3]. Moreover, hepatic lipid accumulation predicts the development of type 2 diabetes independently of other cardiovascular risk factors [4]. Taken together, these observations suggest a causative link between hepatic triacylglycerol content and the development of insulin resistance.

At the intracellular level, the mammalian target of rapamycin (mTOR) integrates insulin receptor (IR) signalling and pathways involved in amino acid/nutrient sensing. Under conditions of acute high-carbohydrate intake, leading to elevated circulating insulin concentrations, mTOR phosphorylation is enhanced via the IR pathway through phosphorylation and activation of a class 1 phosphatidylinositol 3-kinase (PI3K) and protein kinase $\mathrm{B}(\mathrm{PKB} / \mathrm{Akt})$ $[5,6]$. In contrast, under conditions of energy deprivation of cells leading to a decline in cytosolic ATP, an inactive state of mTOR is induced through activation of AMP-activated protein kinase (AMPK) [7]. Activation of mTOR generally results in stimulation of ribosomal protein synthesis, triggered by activation of p70S6 kinase, a downstream target of mTOR. The activity of mTOR is also subject to regulation by other factors, such as leptin and adiponectin, that act via AMPK [8]. Furthermore, activity of the autonomal nervous system affects the metabolic setting in peripheral tissues through as yet poorly characterised molecular mechanisms [9].

A meal-induced increase in plasma leucine concentration represents a strong activator of the mTOR pathway through activation of a class III PI3K-dependent pathway [10]. Furthermore, elevated circulating concentrations of glucose and fatty acids may affect the activity of mTOR $[11,12]$. Hyperglycaemia results in activation of atypical protein kinase $\mathrm{C}$ isoforms that desensitise the IR signalling pathway towards PKB and mTOR [13]. In addition, hyperglycaemia may reduce the expression of genes of the respiratory chain in mitochondria [14], which can feed back to the activation status of mTOR via changes in AMPK activity. Cellular overload with triacylglycerol and/or fattyacid-derived products is highly toxic to mitochondria [1518]. So, both hyperglycaemia and hepatic accumulation of triacylglycerol may feed back to the mTOR/S6K/S6 signalling pathway through changes in AMPK and the insulin receptor signalling pathway.

In the present study, we examined the hepatic activation of $\mathrm{mTOR}$ in relation to insulin resistance and steatosis in two mouse models of hepatic steatosis. In the first model, hepatic steatosis and insulin resistance were induced by 6 weeks of exposure to a high-fat diet. In the second model, acute hepatic steatosis without insulin resistance was acutely induced by pharmacological inhibition of $\beta$-oxidation. Our results indicate that both forms of steatosis were associated with differential effects on mTOR signalling.

\section{Materials and methods}

Animals

The protocol of investigation conformed to the Guide for the Care and Use of Laboratory Animals as published by the NIH (NIH Publication No. 85-23, revised 1996) and was approved by the Institutional Animal Ethical Committee of the Leiden University Medical Center or the Animal Experiments Ethical Commission of the University of Groningen. Male C57BL/6Jico mice (6 weeks old) were purchased from Charles River (Maastricht, the Netherlands) and kept in a 12-h light-dark cycle. The animals were randomly divided in two groups and had free access to water and either standard lab chow or a high-fat diet for 6 weeks.

Diets

The high-fat diet was obtained from Arie Blok Diervoeding (Woerden, the Netherlands, cat no. 4031.05) and consisted (in $\%$ of total energy) of $44 \%$ bovine fat, $19 \%$ protein and $37 \%$ carbohydrate. Chow diet from the same supplier consisted of $32 \%$ protein, 54\% carbohydrate and $14 \%$ crude fat. Both diets were supplemented with vitamins and microelements.

\section{Tetradecylglycidic acid intervention}

Animal experiments were performed as previously described [19]. Tetradecylglycidic acid (TDGA) (inhibitor of carnitine palmitoyltransferase-1 [CPT1]) was suspended in a concentration of $2.0 \mathrm{mg} / \mathrm{ml}$ in a vehicle consisting of $90 \mathrm{mg} / \mathrm{ml}$ BSA in saline [20]. Mice received either $30 \mathrm{mg} / \mathrm{kg}$ TDGA or vehicle by intraperitoneal injection. Food was removed, 
but the mice still had access to water. After $12 \mathrm{~h}$, mice were killed by cardiac puncture under isoflurane anaesthesia. The liver was quickly removed and frozen for further analysis.

Hyperinsulinaemic-euglycaemic clamp

These studies were performed to measure the inhibitory effect of hepatic glucose production in chow-fed $(n=6)$ and high-fat-fed $(n=4)$ mice. After an overnight fast $(15 \mathrm{~h})$, mice were anaesthetised by subcutaneous injection of fentanyl ( $7 \mu \mathrm{l} / \mathrm{g}$ body weight; Hypnorm, Janssen Pharmaceutica, Beerse, Belgium) and midazolam (12.5 mg/g body weight; Genthon, Nijmegen, the Netherlands). After a basal period (60 min), a hyperinsulinaemic-euglycaemic study was started with a primed-continuous infusion of insulin (3.5 $\left.\mathrm{mU} \mathrm{kg}^{-1} \mathrm{~min}^{-1}\right)$ and $\mathrm{D}-\left[{ }^{3} \mathrm{H}\right]$ glucose $\left(2.2 \times 10^{4} \mathrm{~Bq} \mathrm{~kg}^{-1}\right.$ $\min ^{-1}$; adjusted rate $12.5 \%$ for $120 \mathrm{~min}$ to maintain euglycaemia) [21].

Plasma determinations

Insulin was measured by ELISA (Ultrasensitive Mouse Insulin kit; Mercodia, Uppsala, Sweden). Plasma glucose was determined at regular intervals using a glucometer (Accutrend Sensor; Roche Diagnostics, Mannheim, Germany). Leucine concentration in plasma was measured by liquid chromatography as fluorescent derivatives according to manufacturer protocol (AccQ-Fluor; Water, Veenendaal, the Netherlands) optimised for baseline separation of leucine.

\section{Isolation of livers}

Diet-fed mice ( $n=7$ per group) were killed by cervical dislocation following a 1-h infusion with PBS as described under hyperinsulinaemic-euglycaemic clamp [21]. Livers were rapidly removed, snap-frozen in liquid nitrogen and stored at $-80^{\circ} \mathrm{C}$ until further analysis.

Analysis of protein production, phosphorylation and PI3K activity

Livers $(100 \mathrm{mg}$ ) were homogenised in buffer containing (in mmol/l): 30 Tris, 2.5 EDTA, $150 \mathrm{NaCl}, 0.5 \mathrm{Na}_{3} \mathrm{VO}_{4}$, $5 \mathrm{NaF}, 5 \mathrm{MgCl}_{2}, 12.5 \%$ glycerol, $1 \% \mathrm{NP} 40$ and protease inhibitors (Complete; Roche). Lysates were centrifuged $\left(16,100 \mathrm{~g}, 15 \mathrm{~min}, 4^{\circ} \mathrm{C}\right)$ and protein content was determined using a BCA-kit (Pierce, Rockford, IL, USA). Expression and phosphorylation of signalling intermediates were analysed by western blotting. Antibodies for insulin receptor $\beta$-subunit, uncoupling protein (UCP)-2 and UCP3 and phosphotyrosine-horseradish peroxidase conjugate were from SantaCruz Biotechnology (SantaCruz, CA,
USA). Phospho-specific antibodies for PKB/Akt (Ser473), mTOR (Ser2448), AMPK-alpha (Thr172), p70 S6-kinase (Thr421/Ser424), S6 ribosomal protein (Ser235/236) were from Cell Signalling Technology (Beverly, MA, USA). Polyclonal mTOR, IRS-1 and IRS-2 antibodies have been described [22, 23]. PKB/Akt antibodies were kindly provided by B. Burgering (UMC Utrecht, Utrecht, the Netherlands). Cytochrome $\mathrm{C}$ oxidase I and cytochrome C oxidase IV antibodies were from Molecular Probes (Breda, the Netherlands). Tyrosine phosphorylation and associated PI3K-activity were determined in IR, IRS-1- and IRS-2 immunoprecipitates as previously described [24]. Immunoblots and autoradiographs were quantified by ImageGauge software (version 3.12; Fuji Photo Film, Tokyo, Japan).

Analysis of lipid fractions in liver supernatant fractions

Lipids were extracted according to Bligh and Dyer's method [25] and detailed as previously described [26]. In short, $200 \mu \mathrm{g}$ protein of liver supernatant fraction was homogenised in the buffer described above, diluted in $800 \mu \mathrm{l} \mathrm{MilliQ}$, solubilised in $3 \mathrm{ml}$ methanol-chloroform, after which $500 \mu \mathrm{l}$ chloroform, $100 \mu \mathrm{l}$ of internal standard and $1 \mathrm{ml} \mathrm{MilliQ}$ were added. After centrifugation, the chloroform layer was collected and dried. The pellet was dissolved in $50 \mu \mathrm{l}$ chloroform and loaded on a highperformance thin layer chromatography plate. Triacylglycerol and diacylglycerol fractions were normalised by the intensity of internal standard.

Ribosomal protein synthesis

The rate of in vitro translation was measured in an assay using $\left[{ }^{3} \mathrm{H}\right]$ leucine as described in detail [27]. This assay consists of a short-term $\left[{ }^{3} \mathrm{H}\right]$ leucine incorporation of cell lysates, which leads to $\left[{ }^{3} \mathrm{H}\right]$ leucine incorporation predominantly in existing polysomes.

Determination of mitochondrial DNA content

Liver DNA was isolated using the DNeasy kit (Qiagen Benelux, Venlo, the Netherlands). Mitochondrial DNA (mtDNA) content was estimated by real-time PCR by determining the ratio between mtDNA and the $\beta$-actin gene. The primers used for $\beta$-actin were forward $5^{\prime}$ AGAGGGAAATCGTGCGTGAC-3', reverse 5'-CAA TAGTGATGACCTGGCCGT-3', and for mtDNA forward 5'-TTTCATAGGGGCATGAGGAG-3', reverse 5'TGAGGGATGGgTtGTAAGGA-3'. The relative mitochondrial copy number $(\mathrm{Rc})$ was calculated using the following equation: $\mathrm{Rc}=2^{\Delta \mathrm{Ct}}$ and $\Delta=\mathrm{Ct}(\beta$-actin $)-\mathrm{Ct}$ (mtDNA) [28], where Ct is threshold cycle. 
Table 1 Basal characteristics of mice after 6 weeks on chow or high-fat diet

Values are means \pm SEM $p>0.05$ for NS

Cytochrome $\mathrm{C}$ oxidase activity

Cytochrome $\mathrm{C}$ oxidase activity was determined in liver homogenates by measuring the oxidation of cytochrome $\mathrm{C}$ at an absorption of $550 \mathrm{~nm}$. Values were corrected for protein content.

Statistical analysis

All data are presented as mean \pm SEM. Differences between groups were determined by unpaired Student's two-tailed $t$ test using GraphPad Prism analysis software. Significance was accepted at $p<0.05$.

\section{Results}

High-fat diet induces obesity and hepatic insulin resistance but does not affect plasma leucine concentrations in mice

\section{Animal characteristics}

Basal characteristics of chow- and high-fat-fed mice are summarised in Table 1 . Mean body weight was $16 \%$ higher $(p<0.001)$ in high-fat- than in chow-fed mice. Postabsorptive plasma glucose and leucine levels were similar in both groups, whereas postabsorptive plasma insulin levels were slightly higher in mice on a high-fat diet than in chow-fed animals $(p=0.05)$.

High-fat feeding decreased whole-body insulin sensitivity as determined by a hyperinsulinaemic-euglycaemic clamp study (Table 2). Glucose infusion rates required to maintain euglycaemia during the clamp were decreased in high-fat-fed versus chow-fed mice $(p<0.05)$. Furthermore,

Table 2 Clamp characteristics of mice on chow or high-fat diet

\begin{tabular}{llll}
\hline & $\begin{array}{l}\text { Chow } \\
(n=6)\end{array}$ & $\begin{array}{l}\text { High fat } \\
(n=4)\end{array}$ & $\begin{array}{l}p \\
\text { value }\end{array}$ \\
\hline GIR $\left(\mu \mathrm{mol} \mathrm{kg} \mathrm{min}^{-1}\right)$ & $29 \pm 3$ & $21 \pm 3$ & $p<0.05$ \\
HGP basal $\left(\mu \mathrm{mol} \mathrm{kg} \mathrm{min}^{-1}\right)$ & $74 \pm 9$ & $64 \pm 10$ & $\mathrm{NS}$ \\
$\begin{array}{l}\text { HGP hyperinsulinaemic } \\
\left(\mu \mathrm{mol} \mathrm{kg} \mathrm{min}^{-1}\right)\end{array}$ & $34 \pm 4$ & $42 \pm 6$ & $p<0.05$ \\
\hline
\end{tabular}

Values are means \pm SEM. $p>0.05$ for NS. GIR, glucose infusion rate; $H G P$, hepatic glucose production hepatic glucose production during the clamp study was more significantly reduced in the chow-fed mice (from $74 \pm$ $9 \mu \mathrm{mol} \mathrm{kg}{ }^{-1} \mathrm{~min}^{-1}$ at basal to $34 \pm 4 \mu \mathrm{mol} \mathrm{kg}{ }^{-1} \mathrm{~min}^{-1}$ after the clamp) than in the animals on a high-fat diet (from $64 \pm 10$ at basal to $42 \pm 6 \mathrm{mmol} \mathrm{kg} \mathrm{min}^{-1}$ after insulin, $p<0.05$; Table 2).

High-fat feeding, but not induction of hepatic steatosis, induces increased activity of the mTOR-S6 kinase pathway in mouse liver

The phosphorylation of mTOR-Ser2448 was 1.8-fold higher in livers from mice fed a high-fat diet than in those of chow-fed animals ( $p<0.01$; Fig. 1a) and was accompanied by elevated phosphorylation of its downstream targets. Notably, the phosphorylation of p70S6 kinase (Thr421/ Ser424) and its substrate S6 ribosomal protein (Ser235/236) was 2.1- and 2.7-fold higher, respectively, in liver of highfat-fed mice than in that of chow-fed animals (both $p<0.05$; Fig. 1b,c). Protein expression levels of mTOR, p70S6 kinase, and S6 ribosomal protein were similar between mice on a high-fat diet and chow-fed mice (Fig. 1a-c). Protein synthesis was measured at 2.5, 5, 10, 15, and $20 \mathrm{~min}$ after addition of $\left[{ }^{3} \mathrm{H}\right]$ leucine to the cell lysate from chow and high-fat livers. On average, in lysates derived from mice on a high-fat diet, $\left[{ }^{3} \mathrm{H}\right]$ leucine incorporation was increased by $1.4 \pm 0.3$-fold, $p=0.2$. The trend of increased protein synthesis is in line with the sustained activity of the mTOR/S6K pathway. Subsequently, we analysed whether the elevated activity of the mTOR pathway could be ascribed to hepatic steatosis as such. To this end, acute steatosis was induced by inhibition of $\beta$-oxidation with TDGA, leading to a 5-fold increase in hepatic triacylglycerol content [19]. As shown in Fig. 1d-f, TDGA had no significant effect on the phosphorylation of mTOR, p70S6K, and S6 ribosomal protein, suggesting that hepatic steatosis as such does not underlie chronic activation of mTOR signalling.

High-fat diet intervention does not affect the basal phosphorylation of hepatic IR/IRS/PI3K/Akt in mice

We examined whether the slight elevation in plasma insulin levels contributed to the increased activity of the mTOR- 


\section{a}

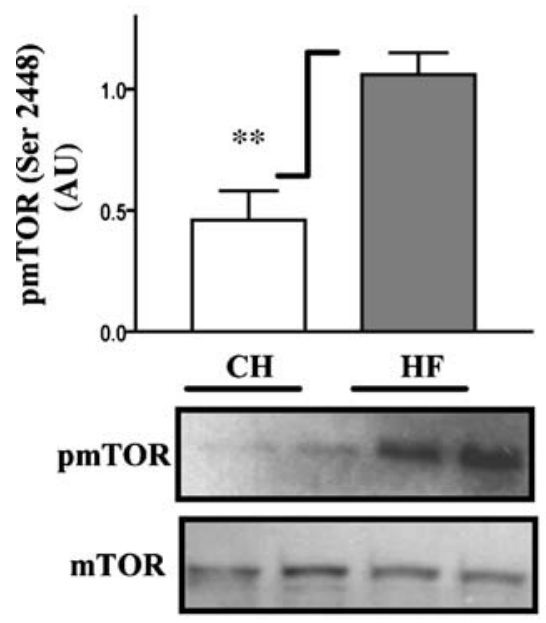

d

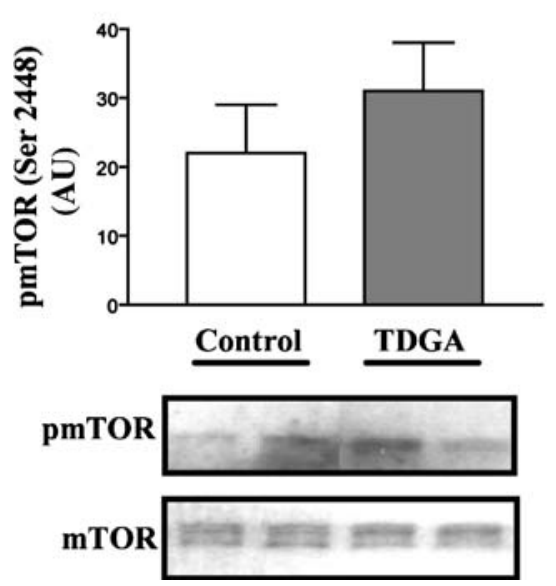

b

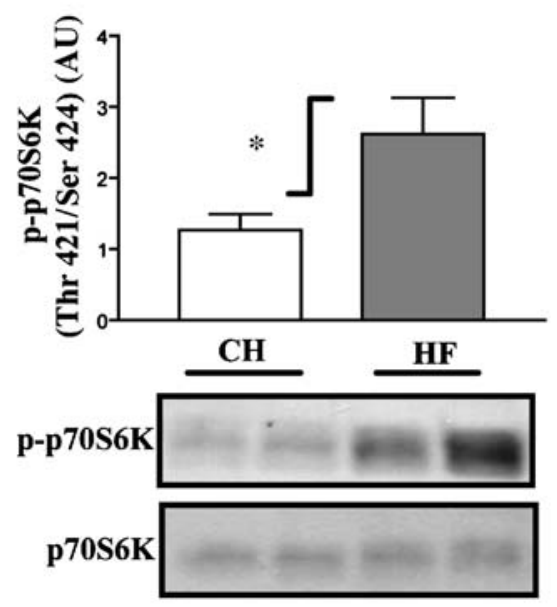

$\mathbf{e}$

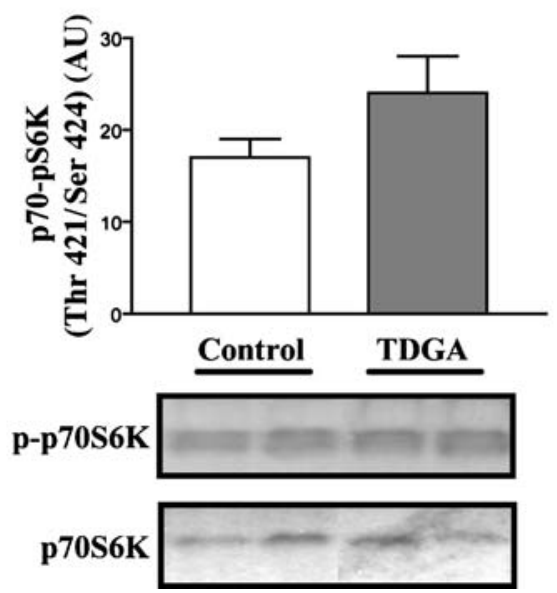

c

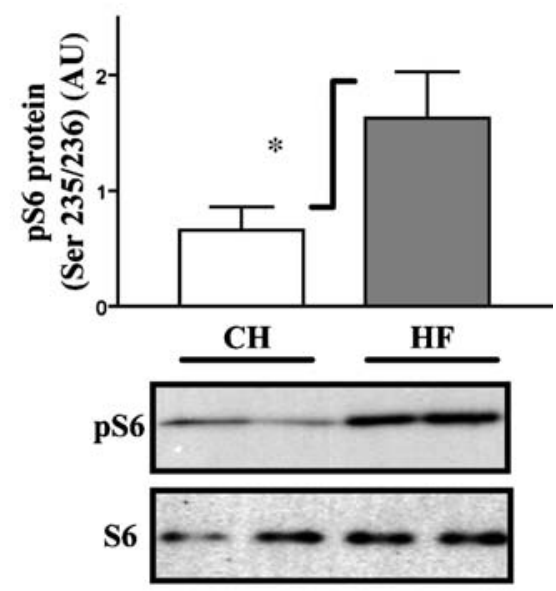

$\mathbf{f}$

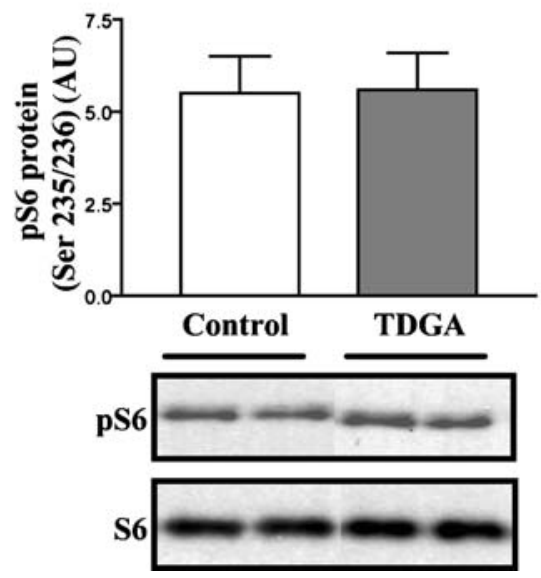

Fig. 1 Hepatic mTOR signalling in chow-fed mice $(\mathrm{CH})$ and mice fed a high-fat (HF) diet (a-c) and in mice injected with the CPT1 inhibitor tetradecylglycidic acid (TDGA) (d-f). Liver lysates $(25-50 \mu \mathrm{g}$ protein) were subjected to immunoblotting with specific antibodies to measure phosphorylation of mTOR (pmTOR) (a, d), p70S6K (pp70S6K) (b, e) and S6 ribosomal protein (pS6) (c, f). After immunoblotting with phospho-specific antibodies, membranes were stripped and re-probed for protein expression. Graphs depict levels of phosphorylated proteins normalised by their expression levels. Each lane represents a sample from an individual mouse. Data are mean \pm SEM, $n=7$ per group. ${ }^{*} p<0.05 ;{ }^{*} p<0.01$. AU, arbitrary units pathway in high-fat-fed mice. Figure $2 \mathrm{a}-\mathrm{c}$ and $\mathrm{g}$ shows that basal tyrosine phosphorylation of the insulin receptor $\beta$ subunit, IRS-1, IRS-2, and Ser473-phosphorylation of PKB/ Akt were similar between mice on a high-fat diet and chowfed mice. Expression levels of these proteins were not affected by the diet intervention (Fig. 2d-g). Accordingly, IRS-1- and IRS-2-associated PI3K activities were similar between the experimental groups (Fig. 2h,i). Therefore, the slightly higher fasting insulin levels in the high-fat-fed animals did not lead to higher basal phosphorylation and activation states of the examined signalling intermediates. From these observations, we conclude that sustained activation of the mTOR/S6K pathway in high-fat-fed animals is unlikely to result from diet-induced alterations in the IR/IRS/PI3K/Akt pathway.

Do alterations in AMPK and mitochondrial function contribute to high-fat diet-induced activation of the mTOR-S6 kinase pathway?

The activation status of mTOR is under control of AMP levels that are sensed by AMPK, in addition to regulation through the IR/IRS/PI3K/Akt pathway. Increased AMP levels are associated with impaired cellular ATP production mediated through reduced mitochondrial oxidative phosphorylation. Fatty acids can fuel mitochondrial activity, but 
also have the ability to disrupt mitochondrial function. Therefore, we examined whether changes occurred in the mitochondrial respiratory chain activity and in the AMPK activation status in mice on a high-fat diet compared with chow-fed mice. No significant change was observed in the phosphorylation status of AMPK between the livers of chow- and high-fat-fed mice (Fig. 3a).

Mitochondrial cytochrome $\mathrm{C}$ oxidase activity was slightly increased in mice on the high-fat diet $(p<0.05)$, while protein expression of subunits I and IV of cytochrome a

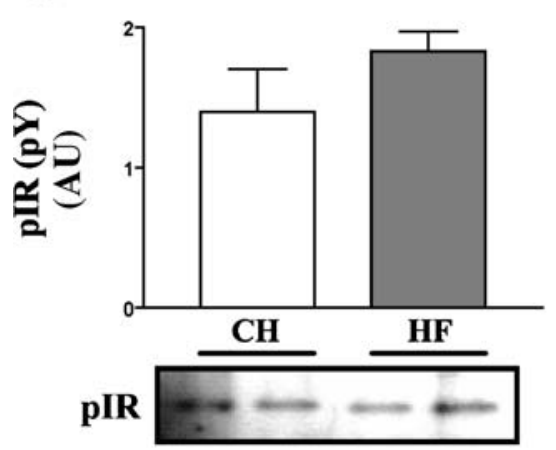

d

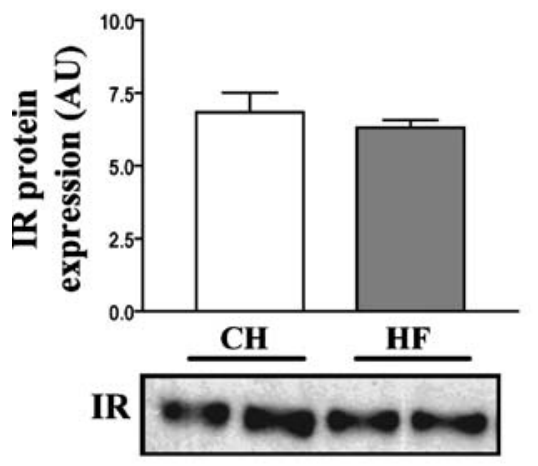

g

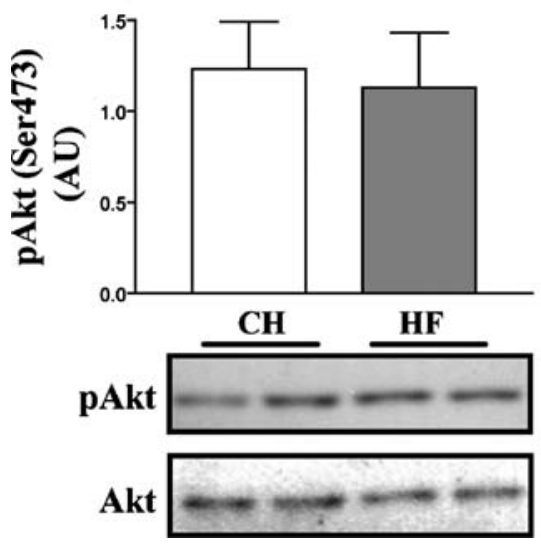

b

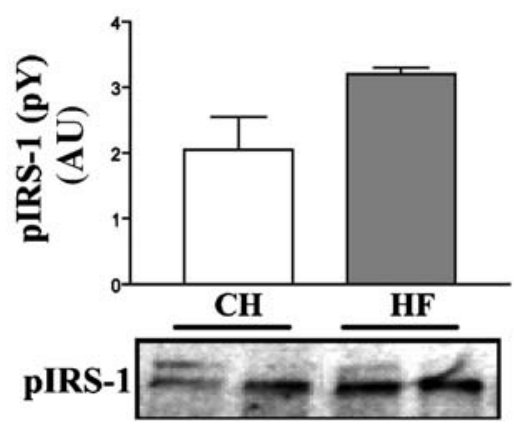

e

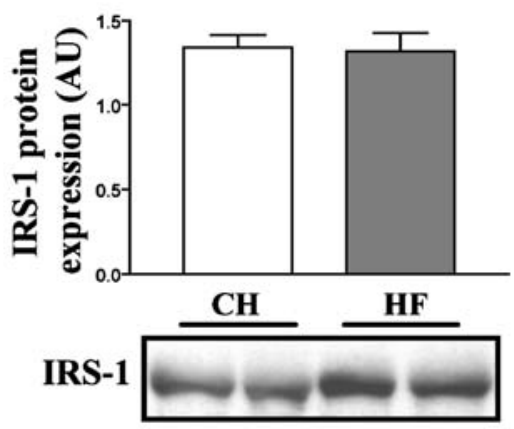

h

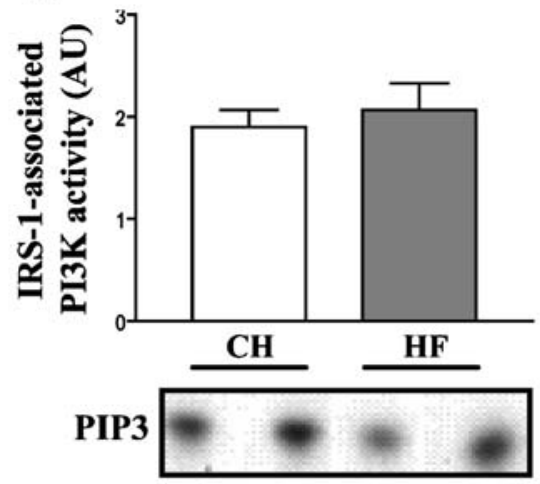

c

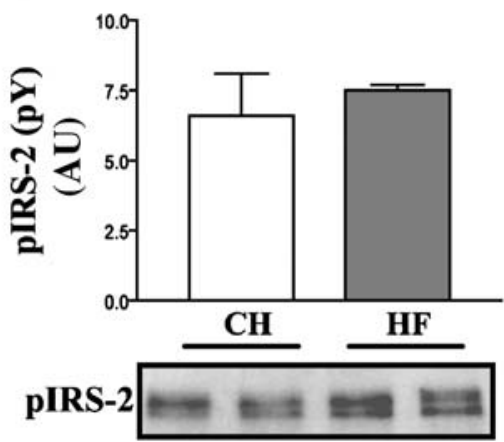

f

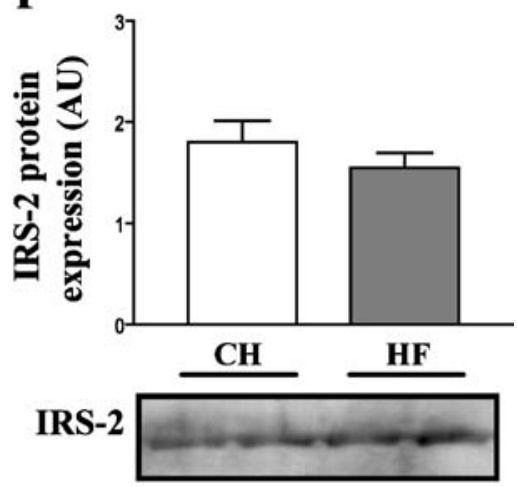

i

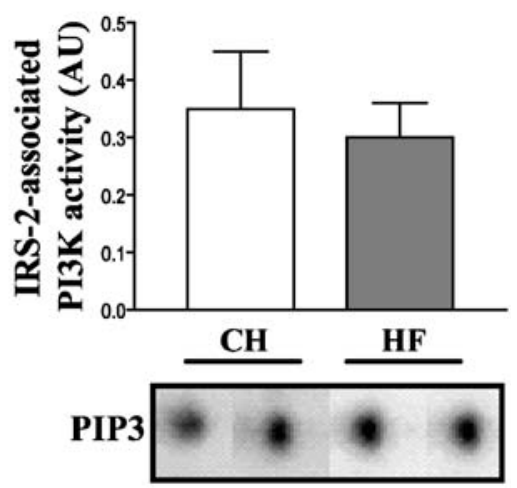

Fig. 2 Hepatic insulin receptor signalling in chow-fed mice $(\mathrm{CH})$ and mice fed a high-fat (HF) diet. Liver lysates (2 $\mathrm{mg}$ protein) were subjected to immunoprecipitation with antibodies against insulin receptor (IR) (a), IRS-1 (b), and IRS-2 (c) and subsequent immunoblotting with phosphotyrosine antibodies (a-d) or lipid kinase assay for measuring PI3K activity $(\mathbf{h}, \mathbf{i})$. Expression of phosphorylated
Akt (pAkt) and total protein expression of IR, IRS-1 and IRS-2 were measured in direct liver lysates using $25 \mu \mathrm{g}$ protein $(\mathbf{d}-\mathbf{g})$. PI3K activities were measured in the presence of PI and ${ }^{32} \mathrm{P}$-labelled-ATP, and phosphatidylinositol 3-phosphate (PIP3) was separated by thin layer chromatography. $\mathbf{h}$, i Representative thin layer chromatography. Data are mean \pm SEM, $n=4-7$ per group. $A U$, arbitrary units 
$\mathrm{C}$ oxidase was unchanged in this group compared with chow-fed mice (Fig. 3b).

Mitochondrial DNA copy number was independent of the nature of the diet (Fig. 3c).

Uncoupling protein 3 is upregulated in human skeletal muscle upon ingestion of a high-fat diet, and this is independent of mitochondrial uncoupling activity [29]. We observed a similar upregulation in the murine liver as UCP3 content was increased twofold in livers of high-fat-fed mice compared with chow-fed mice, while UCP2 protein expression remained unchanged (Fig. 3d).

\section{Discussion}

In this study we analysed the changes induced by a high-fat diet in the basal status of hepatic insulin (IR/IRS/PI3K/Akt) and nutrient (mTOR/S6K/S6) pathways under the condition of hepatic insulin resistance. We show that after 6 weeks on the high-fat diet mice had developed whole-body and hepatic insulin resistance. Under these conditions, we observed a strong, constitutional hepatic activation of mTOR in the postabsorptive state accompanied by increased phosphorylation of known signalling steps downstream of mTOR, such as p70S6K and ribosomal protein S6 as well as increased ribosomal protein synthesis in the liver. In contrast, hepatic accumulation of triacylglycerol, induced by inhibition of $\beta$-oxidation, was not associated
Fig. 3 Hepatic AMPK phosphorylation (a), activity and expression of mitochondrial (mt) proteins $(\mathbf{b}, \mathbf{d})$ and mtDNA (c) in chow-fed mice $(\mathrm{CH})$ and mice fed a high-fat (HF) diet. Liver lysates $(25 \mu \mathrm{g}$ protein) were subjected to western blotting with antibodies against phospho-AMPK (pAMPK) (a), subunits I and IV of complex IV of cytochrome $\mathrm{C}$ oxidase (COX) (b), and uncoupling protein (UCP)-2 and UCP3 (d). COX activity was determined by oxidation of cytochrome $\mathrm{C}$ and normalised for protein amount (b). mtDNA was quantified by real-time PCR and normalised by expression of $\beta$-actin. Data are mean \pm SEM, $n=7$ per group. ${ }^{*} p<0.05$. $A U$, arbitrary units; $R c$, relative mitochondrial number
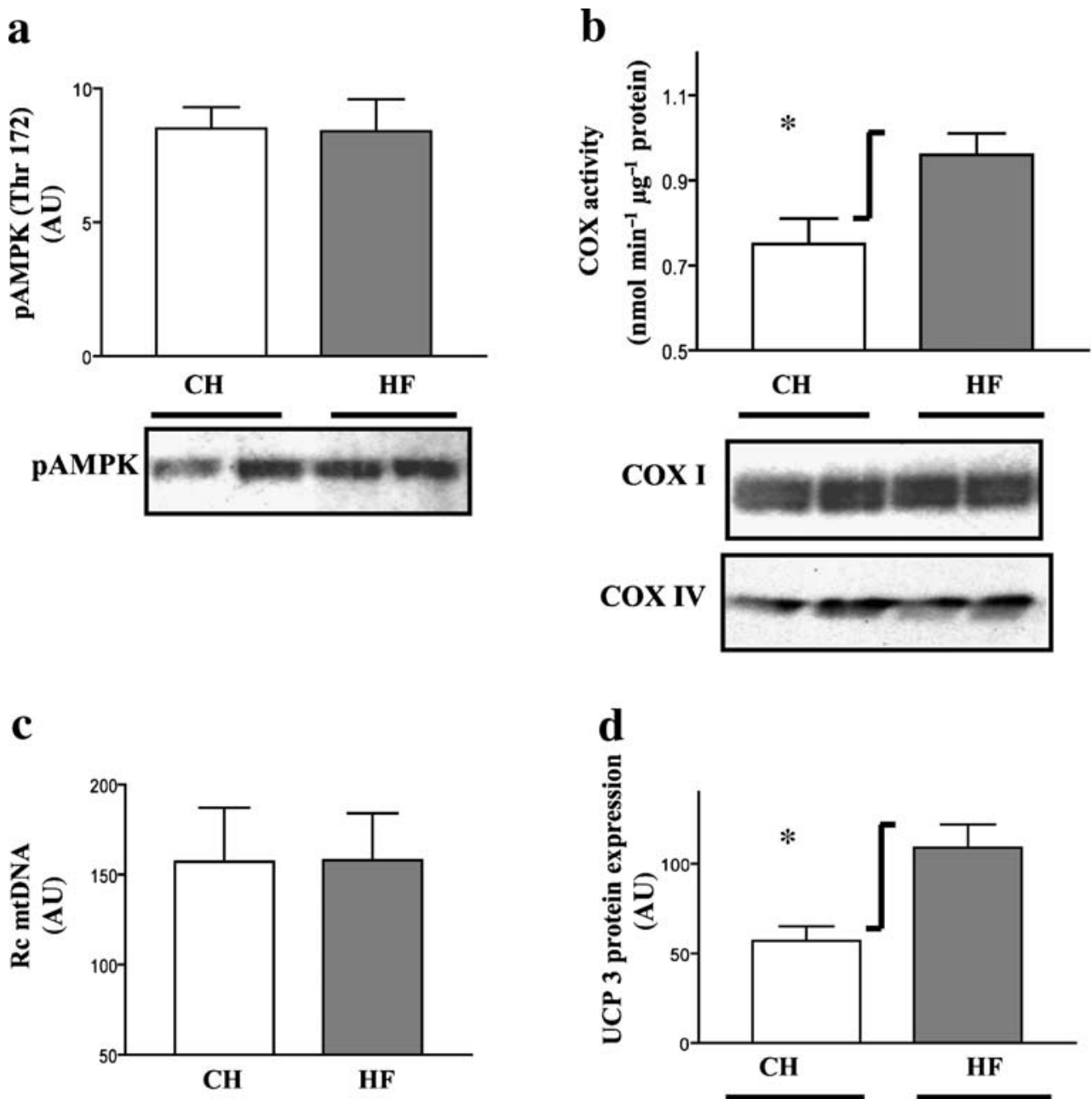

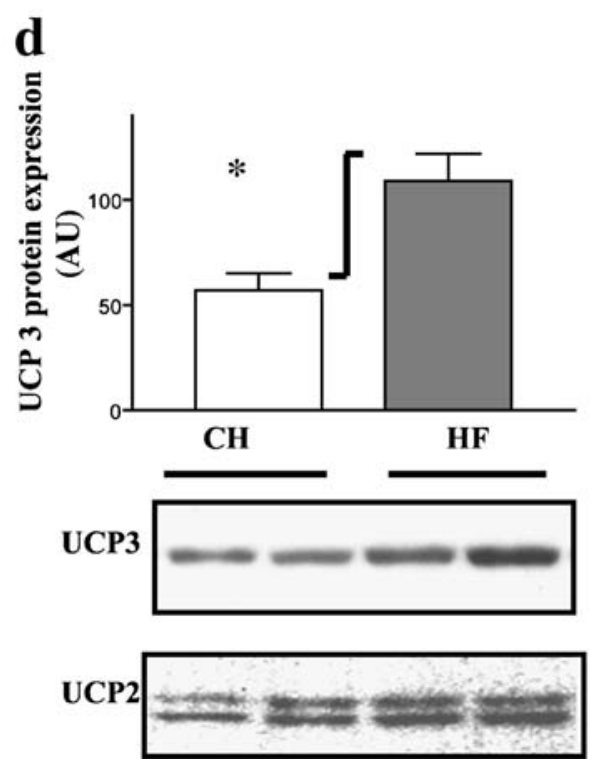


with hepatic insulin resistance or activation of the mTOR pathway. Therefore, hepatic insulin resistance, but not hepatic steatosis as such, associates with sustained activation of the mTOR nutrient sensing pathway.

Various metabolic factors can modulate mTOR phosphorylation [30], and we therefore investigated whether changes in these known factors upon high-fat feeding could account for the sustained activity of the mTOR pathway. Some amino acids, especially leucine, are strong activators of mTOR activity [31,32]. The precise sensors involved in amino acid-induced mTOR activation are poorly understood in higher eukaryotes. A recent study suggests that class 3 PI3K (hVps15) rather than class 1 PI3K may mediate the effect of amino acids. Class 1 PI $3 \mathrm{~K}$ is the isoform that undergoes activation through the insulin receptor [10]. However, plasma leucine concentrations were not different between animals on a high-fat diet compared with chow-fed mice, suggesting that circulating leucine was not responsible for the sustained mTOR activation.

Two additional signalling pathways have been identified that contribute to mTOR activation. One pathway involves the insulin/IGF-1 receptor, IRS-proteins and PI3K, leading to activation of $\mathrm{Akt} / \mathrm{PKB}$, which is known to phosphorylate and activate mTOR [10]. The other pathway regulating mTOR activity involves AMPK. Under conditions of energy depletion, e.g. due to mitochondrial damage through overload of cells with fatty acids, the resulting increase in cytosolic AMP concentrations activates AMPK. This, in turn, leads to inactivation of mTOR by preventing the activation of a TOR kinase that is controlled by the guanosine-triphosphate-bound status of Rheb [14, 33, 34]. Therefore, to evaluate whether the high-fat diet enhances the activation status of mTOR through changes in IR signalling or AMPK activity, we analysed the activation status of these pathways in the liver of high-fat-fed mice. No significant change in the activation status of the IR, class $1 \mathrm{PI} 3 \mathrm{~K}$ and $\mathrm{PKB}$ was seen that could explain the enhanced phosphorylation of mTOR in the animals on a high-fat diet. When analysing AMPK activity and the mitochondrial activity that controls AMPK through ATP production, we observed no change in AMPK phosphorylation, mitochondrial DNA copy number and expression levels of subunits I and IV of cytochrome C oxidase, whereas a slight increase in overall cytochrome $\mathrm{C}$ activity was seen in high-fat-fed compared with chow-fed animals. These findings indicate that the hepatocytes in animals fed a high-fat diet are not ATP-deficient as a result of a fatty acid-induced mitochondrial dysfunction.

It was recently reported that a high-fat diet induces expression of mitochondrial UCP3 in skeletal muscle [29]. Rather than being an uncoupler of the respiratory chain, this protein may protect mitochondria against fatty acid over- load. We observed in the livers of the high-fat mice a twofold increase in UCP3 protein content compared with the chow-fed mice. However, UCP3 protein expression did not change upon CPT1 inhibition in the liver (results not shown). These data point to a sensing mechanism for highfat feeding, which operates in muscle and liver and regulates the expression level of UCP3. This sensing mechanism may also contribute to the association between high-fat feeding and mTOR activation and the development of insulin resistance.

High-fat diet-induced hepatic steatosis and hepatic insulin resistance are closely associated phenomena. The observed situation of sustained mTOR activation in response to the high-fat diet could be directly related to high hepatic triacylglycerol content, e.g. through triacylglycerol/NEFA-induced changes of hepatic signalling. Therefore, we examined whether hepatic steatosis as such, without concomitant insulin resistance, is sufficient to induce the sustained activation of the nutritional pathway. For this purpose, we analysed mTOR activation in mice treated with TDGA, an inhibitor of fatty acid $\beta$-oxidation. TDGA treatment in combination with fasting results in acute hepatic steatosis uncoupled from insulin resistance $[19,35]$. Remarkably, no change in hepatic mTOR activation was seen in these mice, suggesting that other factors than intrahepatic triacylglycerol accumulation are responsible for the induction of sustained mTOR activation in high-fat-fed mice. One option to explain the differences in phenotype could be a difference in the ratio of triacylglycerol: diacylglycerol in liver, as diacylglycerol is a known potent activator of various protein kinase $\mathrm{C}$ isoforms. The activation of protein kinase $\mathrm{C}$ could be a modulator of the phenotype. However, analysis of triacylglycerol:diacylglycerol ratios showed that in all animals fatty acids were predominantly present in the triacylglycerol pool and that diacylglycerol levels were present in amounts around the detection limit of the assay (4-5\% of triacylglycerol levels). High-fat-induced adipokine profiles or reprogramming of particular brain nuclei are plausible factors. The recent observation by Khamzina et al. [36] that obese rats exhibit sustained mTOR/S6K activation in multiple tissues is also compatible with a situation that circulating or neuronal factors are involved in mTOR activation in multiple tissues.

Taken together, our results show that in the murine liver the activity of the mTOR-controlled pathway is enhanced in response to insulin resistance induced by high-fat feeding. This activation proceeds through an as yet unidentified sensor, which does not involve sustained activation of the insulin receptor pathway, changes in AMPK activity or circulating leucine levels. Furthermore, hepatic steatosis alone, without the development of insulin resistance, is not sufficient to activate this pathway, showing that triacylglycerol itself is unlikely to be the mTOR-activating agent. 
Acknowledgements We would like to thank P. van den Broek, A. C. Heijboer and A. van den Hoek for their valuable help. We thank I. Que and P. Modderman for their excellent technical assistance. The financial support of the Dutch Diabetes Research Foundation (grant no. 2002.00.32) is gratefully acknowledged.

Duality of interest This study was supported by the Dutch Diabetes Research Foundation (to J.A. Maassen, J.A. Romijn, L.M. Havekes). The authors have stated that they have no duality of interest.

\section{References}

1. Yki-Jarvinen H, Westerbacka J (2005) The fatty liver and insulin resistance. Curr Mol Med 5:287-295

2. Boden G, Chen X, Capulong E, Mozzoli M (2001) Effects of free fatty acids on gluconeogenesis and autoregulation of glucose production in type 2 diabetes. Diabetes 50:810-816

3. Weyer C, Bogardus C, Pratley RE (1999) Metabolic characteristics of individuals with impaired fasting glucose and/or impaired glucose tolerance. Diabetes 48:2197-2203

4. Lautamaki R, Borra R, Iozzo P et al (2006) Liver steatosis coexists with myocardial insulin resistance and coronary dysfunction in patients with type 2 diabetes. Am J Physiol Endocrinol Metab 291:E282-E290

5. Scott PH, Brunn GJ, Kohn AD, Roth RA, Lawrence JC Jr (1998) Evidence of insulin-stimulated phosphorylation and activation of the mammalian target of rapamycin mediated by a protein kinase B signaling pathway. Proc Natl Acad Sci USA 95:7772-7777

6. Shamji AF, Nghiem P, Schreiber SL (2003) Integration of growth factor and nutrient signaling: implications for cancer biology. Mol Cell 12:271-280

7. Tokunaga C, Yoshino K, Yonezawa K (2004) mTOR integrates amino acid- and energy-sensing pathways. Biochem Biophys Res Commun 313:443-446

8. Kahn BB, Alquier T, Carling D, Hardie DG (2005) AMPactivated protein kinase: ancient energy gauge provides clues to modern understanding of metabolism. Cell Metab 1:15-25

9. Kreier F, Kap YS, Mettenleiter TC et al (2006) Tracing from fat tissue, liver, and pancreas: a neuroanatomical framework for the role of the brain in type 2 diabetes. Endocrinology 147:1140-1147

10. Nobukuni T, Joaquin M, Roccio M et al (2005) Amino acids mediate $\mathrm{mTOR} /$ raptor signaling through activation of class 3 phosphatidylinositol 3OH-kinase. Proc Natl Acad Sci USA 102:14238-14243

11. Pirola L, Johnston AM, Van Obberghen E (2004) Modulation of insulin action. Diabetologia 47:170-184

12. Nguyen MT, Satoh H, Favelyukis S et al (2005) JNK and tumor necrosis factor- $\alpha$ mediate free fatty acid-induced insulin resistance in 3T3-L1 adipocytes. J Biol Chem 280:35361-35371

13. Kroder G, Bossenmaier B, Kellerer M et al (1996) Tumor necrosis factor-alpha- and hyperglycemia-induced insulin resistance. Evidence for different mechanisms and different effects on insulin signaling. J Clin Invest 97:1471-1477

14. Patti ME, Kahn BB (2004) Nutrient sensor links obesity with diabetes risk. Nat Med 10:1049-1050

15. Farrell GC (2002) Drugs and steatohepatitis. Semin Liver Dis 22:185-194

16. Sanyal AJ, Campbell-Sargent C, Mirshahi F et al (2001) Nonalcoholic steatohepatitis: association of insulin resistance and mitochondrial abnormalities. Gastroenterology 120:11831192

17. Fromenty B, Pessayre D (1997) Impaired mitochondrial function in microvesicular steatosis. Effects of drugs, ethanol, hormones and cytokines. J Hepatol 26:43-53
18. Bremer J, Norum KR (1982) Metabolism of very long-chain monounsaturated fatty acids (22:1) and the adaptation to their presence in the diet. J Lipid Res 23:243-256

19. Grefhorst A, Hoekstra J, Derks TG et al (2005) Acute hepatic steatosis in mice by blocking beta-oxidation does not reduce insulin sensitivity of very-low-density lipoprotein production. Am J Physiol Gastrointest Liver Physiol 289:G592-G598

20. Wolkowicz PE, Urthaler F, Forrest C et al (1999) 2-Tetradecylglycidic acid, an inhibitor of carnitine palmitoyltransferase-1, induces myocardial hypertrophy via the AT1 receptor. J Mol Cell Cardiol 31:1405-1412

21. Voshol PJ, Haemmerle G, Ouwens DM et al (2003) Increased hepatic insulin sensitivity together with decreased hepatic triglyceride stores in hormone-sensitive lipase-deficient mice. Endocrinology 144:3456-3462

22. Kessler A, Uphues I, Ouwens DM, Till M, Eckel J (2001) Diversification of cardiac insulin signaling involves the p85 alpha/ beta subunits of phosphatidylinositol 3-kinase. Am J Physiol Endocrinol Metab 280:E65-E74

23. Withers DJ, Ouwens DM, Nave BT et al (1997) Expression, enzyme activity, and subcellular localization of mammalian target of rapamycin in insulin-responsive cells. Biochem Biophys Res Commun 241:704-709

24. Ouwens DM, Boer C, Fodor M et al (2005) Cardiac dysfunction induced by high-fat diet is associated with altered myocardial insulin signalling in rats. Diabetologia 48:1229-1237

25. Bligh EG, Dyer WJ (1959) A rapid method of total lipid extraction and purification. Can J Biochem Physiol 37:911-917

26. Heijboer AC, Voshol PJ, Donga E et al (2005) High fat diet induced hepatic insulin resistance is not related to changes in hypothalamic mRNA expression of NPY, AgRP, POMC and CART in mice. Peptides 26:2554-2558

27. Brandsma M, Janssen GM, Moller W (1997) Termination of quiescence in Crustacea. The role of transfer RNA aminoacylation in the brine shrimp Artemia. J Biol Chem 272:2891228917

28. Szuhai K, Ouweland J, Dirks R et al (2001) Simultaneous A8344G heteroplasmy and mitochondrial DNA copy number quantification in myoclonus epilepsy and ragged-red fibers (MERRF) syndrome by a multiplex molecular beacon based real-time fluorescence PCR. Nucleic Acids Res 29:E13

29. Hesselink MK, Mensink M, Schrauwen P (2003) Human uncoupling protein-3 and obesity: an update. Obes Res 11: 1429-1443

30. Kwon G, Marshall CA, Pappan KL, Remedi MS, McDaniel ML (2004) Signaling elements involved in the metabolic regulation of mTOR by nutrients, incretins, and growth factors in islets. Diabetes 53(Suppl 3):S225-S232

31. Nair KS, Short KR (2005) Hormonal and signaling role of branched-chain amino acids. J Nutr 135:1547S-1552S

32. Deldicque L, Theisen D, Francaux M (2005) Regulation of mTOR by amino acids and resistance exercise in skeletal muscle. Eur J Appl Physiol 94:1-10

33. Sarbassov DD, Ali SM, Sabatini DM (2005) Growing roles for the mTOR pathway. Curr Opin Cell Biol 17:596-603

34. Martin DE, Hall MN (2005) The expanding TOR signaling network. Curr Opin Cell Biol 17:158-166

35. Duivenvoorden I, Teusink B, Rensen PC et al (2005) Acute inhibition of hepatic beta-oxidation in $\mathrm{APOE}^{*} 3$ Leiden mice does not affect hepatic VLDL secretion or insulin sensitivity. J Lipid Res 46:988-993

36. Khamzina L, Veilleux A, Bergeron S, Marette A (2005) Increased activation of the mammalian target of rapamycin pathway in liver and skeletal muscle of obese rats: possible involvement in obesity-linked insulin resistance. Endocrinology 146:1473-1481 